\title{
Plantas medicinais da Bacia do Rio das Velhas: avaliação das condições para produção e uso em saúde pública
}

RICARDO, L.M. ${ }^{12}$; GOULART, E.M.A. ${ }^{3}$; BRANDÃO, M.G.L..$^{12^{*}}$

'Banco de Dados e Amostras de Plantas Aromáticas, Medicinais e Tóxicas, Museu de História Natural e Jardim Botânico, Universidade Federal de Minas Gerais (UFMG) - Av. Gustavo da Silveira, 1035. Belo Horizonte (MG) - Brasil. CEP: 31010-010,; '2Faculdade de Farmácia / UFMG - Av. Antônio Carlos, 6627, Pampulha. Belo

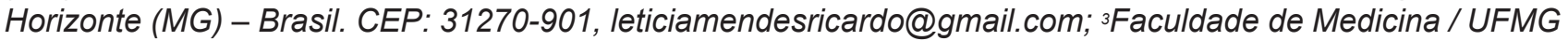
- Av. Alfredo Balena, 190. Belo Horizonte (MG) - Brasil. CEP: 30130-100.*Autor para correspondência: mbrandao@ufmg.br

RESUMO: O desenvolvimento de projetos que contemplem o cultivo e beneficiamento de plantas medicinais, com qualidade, é urgente e necessário, especialmente quando se considera a expansão da oferta desses produtos para o Sistema Único de Saúde (SUS). Nesta pesquisa, foi avaliada a existência de iniciativas que produzem e disponibilizam plantas medicinais de interesse do SUS, em municípios que compõem a Bacia Hidrográfica do Rio das Velhas (BHRV), em Minas Gerais. Foram visitados 45 municípios da Bacia buscando informações sobre atividades já existentes de produção e uso coletivo de plantas medicinais. Os dados sobre plantas medicinais já existentes foram posteriormente confrontados com informações epidemiológicas, como as principais causas de internações hospitalares e cobertura de equipes da Estratégia da Saúde da Família (ESF). A pesquisa evidenciou a existência de atividades filantrópicas e comerciais, relativas à utilização coletiva de plantas medicinais, apenas em Belo Horizonte, Capim Branco, Curvelo, Lassance, Nova Lima e Sete Lagoas. Vinte e sete espécies, nativas e exóticas, presentes na RDC 10/2010 da Anvisa são produzidas nessas iniciativas, e elas ocorrem tanto de forma espontânea como por meio de cultivo. A correlação das principais causas de internação hospitalar com as espécies vegetais disponíveis revelou potenciais locais de aproveitamento das plantas, inclusive pela ESF. O desenvolvimento da cadeia produtiva, trabalhando desde o cultivo até a dispensação aos usuários do SUS, pode representar uma oportunidade de integração de diferentes atores e instituições da região, além de incrementar o desenvolvimento econômico-social e a preservação da biodiversidade local.

Palavras-chave: Rio das Velhas, Sistema Único de Saúde, Cadeia produtiva de plantas medicinais.

\begin{abstract}
Medicinal plants of the Rio das Velhas watershed region: potential for production and use in public health. The development of projects that include the cultivation and processing of medicinal plants with quality is urgent and necessary, especially when considering the offer increase of these products to the Unified Health System (SUS). In this study, we evaluated the potential of the Rio das Velhas' watershed region (BHRV) in producing and using medicinal plants. We visited 45 cities of the watershed, seeking information about existing production and collective use of medicinal plants. Afterwards, the data obtained in the fieldwork were confronted with epidemiological information, such as hospitalization rates and coverage of the Family Health Strategy. The research showed the existence of commercial and philanthropic activities in Belo Horizonte, Capim Branco, Curvelo, Lassance, Nova Lima and Sete Lagoas. Twenty-seven species, native and exotic ones, included in the 10/2010 Resolution edited by the Brazilian Health Surveillance Agency (Anvisa), are produced in these initiatives, and they occur both spontaneously and through cultivation. The correlation of the leading causes for hospitalization and the available plant species showed potential use of local plants, including by the Family Health Strategy. The development of the production chain, from the plants' cultivation to the medicines' dispensation for the SUS users, may represent an opportunity of integration of different actors and institutions in the region, besides increasing the economic and social development and contributing to the preservation of local biodiversity.
\end{abstract}

Keywords: Rio das Velhas, Unified Health System, Medicinal plants chain production.

Recebido para publicação em 01/04/2013

Aceito para publicação em 06/08/2014

10.1590/1983-084X/13_004

Rev. Bras. PI. Med., Campinas, v.17, n.3, p.398-406, 2015. 


\section{INTRODUÇÃO}

Desde a década de 1970, a Organização Mundial da Saúde (OMS) vem reconhecendo a importância das plantas medicinais como recurso terapêutico e estimulando o desenvolvimento de pesquisas que as transformem em produtos com eficácia, segurança e qualidade. De fato, somente assim é possível que o uso das plantas aconteça de forma coletiva dentro de programas de saúde pública (OMS, 1979; 2000; 2011). Muitas plantas utilizadas na medicina tradicional de vários países já foram submetidas a estudos e suas ações farmacológicas foram confirmadas. Já outras espécies, apesar de contarem com o amplo emprego por determinadas populações, não tiveram seus efeitos confirmados e ainda mostraram-se tóxicas. Apesar da vasta flora medicinal disponível e dos desenvolvimentos técnico-científicos na área, as plantas medicinais nativas ainda são pouco estudadas e aproveitadas no Brasil (Brandão et al., 2009; Calixto, 2005). Além disto, vários estudos mostram que, mesmo produtos preparados com plantas exóticas e importadas, que contam com estudos de eficácia e segurança estabelecidos, não vêm sendo disponibilizados no Brasil com qualidade suficiente para uso como fitoterápico (Brandão et al., 2013; Martins \& Brandão, 2006; Brandão et al., 2002). Neste contexto, a produção de matéria-prima de qualidade, com teores adequados de princípios ativos e ausência de adulterações e contaminações, especialmente daquelas espécies já estudadas ou priorizadas pelos órgãos governamentais, torna-se urgente e necessária (OMS, 2011).

Em 2006, o governo federal aprovou a Política Nacional de Plantas Medicinais e Fitoterápicos (Brasil, 2006a), que foi seguida pelo Programa Nacional de Plantas Medicinais e Fitoterápicos (PNPMF) (Brasil, 2008a). O Programa tem seus fundamentos na Política Nacional, e definiu como princípios orientadores: (i) a ampliação das opções terapêuticas e melhoria da atenção à saúde aos usuários do Sistema Único de Saúde (SUS); (ii) o uso sustentável da biodiversidade brasileira; (iii) a valorização e preservação do conhecimento tradicional das comunidades e povos tradicionais; (iv) o fortalecimento da agricultura familiar; (v) o crescimento com geração de emprego e renda, redutor das desigualdades regionais; (vi) o desenvolvimento tecnológico e industrial; (vii) a inclusão social e redução das desigualdades sociais e; (viii) a participação popular e controle social. Uma maneira de se trabalhar tais princípios, de forma integral e articulada, é por meio do fortalecimento das cadeias e arranjos produtivos locais (APL), possibilitando a inserção socioeconômica das populações de territórios caracterizados pelo baixo dinamismo econômico, além de indicadores sociais precários.

É fato conhecido que muitas atividades relacionadas à produção e distribuição de plantas medicinais, públicas, filantrópicas ou comerciais, acontecem em várias partes do Brasil. Essas iniciativas podem representar um ponto de partida importante para o desenvolvimento da cadeia produtiva de plantas medicinais no âmbito do SUS. Nesta pesquisa, foram avaliadas as condições nas quais iniciativas que produzem e disponibilizam plantas medicinais de interesse do SUS acontecem em municípios que compõem a Bacia Hidrográfica do Rio das Velhas (BHRV), em Minas Gerais. Esta região foi selecionada para o estudo por ser a sede do Projeto Manuelzão de revitalização da Bacia do Rio das Velhas, desenvolvido pela Faculdade de Medicina da UFMG. O projeto desenvolve várias atividades com a interface saúde versus meio ambiente, estando a população já bastante sensibilizada a esses temas. Além disto, a região abrange importantes biomas brasileiros, ricos em espécies nativas com amplo histórico de uso na medicina tradicional.

\section{MÉTODO \\ Área estudada}

A BHRV está localizada na região central do estado de Minas Gerais, entre as coordenadas $171^{\prime} 5^{\prime}$ e $20^{\circ} 25^{\prime} \mathrm{S}-43^{\circ} 25^{\prime}$ e $44^{\circ} 50^{\prime} \mathrm{W}$, apresentando uma forma alongada na direção norte-sul (Figura 1).

O Rio das Velhas é o maior afluente em extensão do Rio São Francisco, com 801 km. Ele nasce no município de Ouro Preto, dentro do Parque Municipal das Andorinhas, e deságua no Rio São Francisco, no distrito de Barra do Guaicui, município de Várzea da Palma. A BHRV é subdividida em Alto, Médio e Baixo Rio das Velhas. O Alto Rio das Velhas compreende toda a região denominada Quadrilátero Ferrífero, tendo o Município de Ouro Preto como o limite ao sul e os municípios de Belo Horizonte, Contagem e Sabará como limite ao norte. Esta região é caracterizada por uma elevada altitude, sendo a vegetação nativa composta por espécies de transição entre Mata Atlântica e o Cerrado. O médio Rio das Velhas atravessa o município de Curvelo e, em outro trecho, coincide com os limites do município de Corinto, tendo a vegetação nativa caracterizada pelo cerrado. Já o baixo Rio das Velhas compreende, ao sul, a linha divisória entre os municípios de Monjolos, Gouveia e Presidente Kubitscheck e, ao norte, os municípios de Buenópolis, Joaquim Felício, Várzea da Palma e Pirapora. Nesta região, a vegetação é caracterizada pelo cerrado e alguns campos de altitude.

A população da BHRV, estimada em

Rev. Bras. PI. Med., Campinas, v.17, n.3, p.398-406, 2015. 


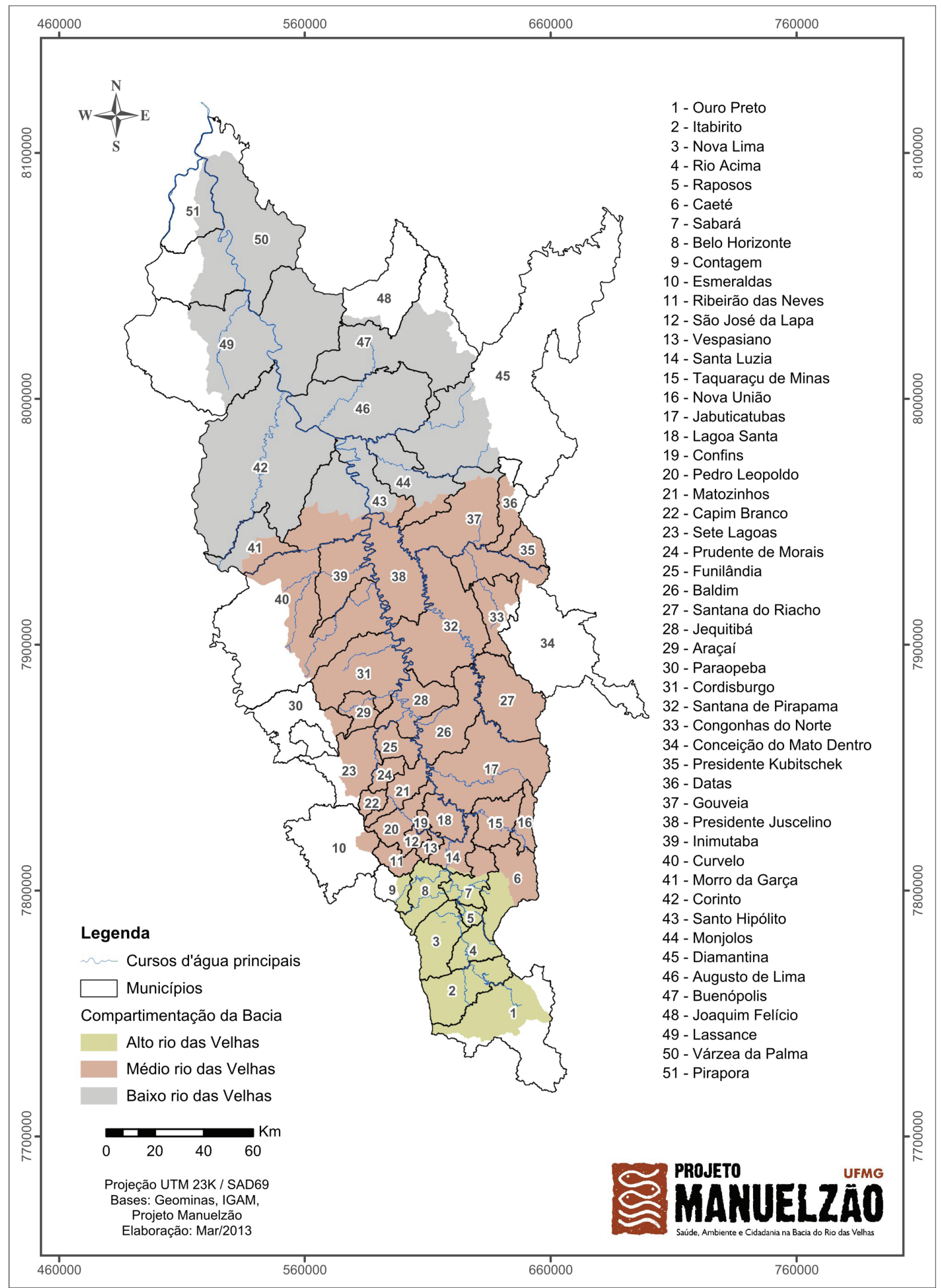

FIGURA 1. Localização da Bacia Hidrográfica Rio das Velhas 
4,6 milhões de habitantes (IBGE, 2010), está distribuída nos 51 municípios banhados pelo rio e seus afluentes. Esses municípios têm uma importância econômica (42\% do PIB mineiro) e social significativa, devido à sua localização que inclui a maior parte da Região Metropolitana de Belo Horizonte (RMBH).

\section{Visita às iniciativas de produção/ distribuição de plantas medicinais nos municípios da BHRV \\ Dos 51 municípios que compõem a BHRV,} 45 foram visitados entre março de 2010 e dezembro de 2012 (não foi possível visitar os demais devido à dificuldade de acesso), em busca de informações sobre as condições nas quais acontecem atividades públicas, filantrópicas ou comerciais, relacionada à produção e distribuição de plantas medicinais. Foi feita uma avalição observacional considerando aspectos como a ocorrência, natural ou cultivada, das espécies listadas na Resolução de Diretoria Colegiada (RDC) $n^{\circ} 10$, de 10 de março de 2010, da Anvisa (Brasil, 2010). Esta lista traz uma relação de 66 espécies medicinais consideradas prioritárias para aquele órgão para a preparação de fitoterápicos, ou seja, infusões, decocções e macerações destinadas ao uso episódico, oral ou tópico para o alívio sintomático de algumas doenças. Foi avaliado também se havia maior produção (cultivo) de determinada espécie, além de infraestrutura disponível para secagem, armazenagem e distribuição.

Após a identificação de cada produtor, as plantas da RDC de ocorrência mais frequente foram registradas, geo-referenciadas, coletadas e encaminhadas para identificação taxonômica (exsicatas) e/ou estudos de autenticidade em laboratório (depositadas no Banco de Dados e Amostras de Plantas Aromáticas, Medicinais e Tóxicas - DATAPLAMT/UFMG, registros para as espécies nativas DAT 2012 a DAT 228).

\section{Levantamento de normas regulatórias para as demais plantas medicinais \\ Para as demais plantas identificadas na} região, foi observada a existência de referências em diferentes publicações do governo brasileiro. As plantas foram checadas na lista de medicamentos fitoterápicos de registro simplificado da Anvisa, constante na Instrução Normativa (IN) $n^{\circ} 05$, de 11 de dezembro de 2008 (Brasil, 2008b) e na lista de espécies de interesse do SUS, Renisus, do Ministério da Saúde (Brasil, 2009). O cruzamento das informações sobre as plantas que ocorrem na Bacia do Rio das Velhas com essas normas é importante, por se tratar de espécies já consideradas prioritárias pelos órgãos que elaboram políticas nacionais, e fazem a regulação e o controle sanitário no país.

Levantamento de dados epidemiológicos Foram levantados, por meio do Datasus (Brasil, 2013a), informações sobre a cobertura populacional de equipes da Estratégia Saúde da Família (ESF) em 2010. Foram coletados também o número e principais causas de internações em cada município, segundo a Classificação Estatística Internacional de Doenças e Problemas Relacionados à Saúde 10ª edição (CID-10) (Brasil, 2013b).

\section{RESULTADO E DISCUSSÃO}

Neste estudo, foram visitados 45 municípios da BHRV buscando identificar iniciativas de produção e disponibilização de plantas medicinais já em funcionamento, e verificar o potencial delas em produzir matéria-prima vegetal de qualidade para o SUS. A despeito da extensa área geográfica abrangida pela BHRV e o número elevado da população, apenas seis municípios contam com alguma atividade regular relacionada à produção $\mathrm{e}$ distribuição de plantas medicinais. Essas atividades acontecem em Belo Horizonte (Parque Municipal Burle Marx), Capim Branco (Sociedade Espírita Maria Nunes), Curvelo (Ervanaria Herbarium), Lassance (produtor do Empório do Cerrado), Nova Lima (Instituto Kairós) e em Sete Lagoas (Indústria Textil Cedro). Em todos esses locais são cultivadas plantas medicinais que são distribuídas, gratuitamente ou não, para a comunidade. Vinte e sete espécies de plantas, nativas e exóticas, presentes na RDC 10 da Anvisa, são encontradas mais frequentemente nos locais visitados (Tabelas 1 e 2).

Por meio das causas de internações hospitalares por local de residência é possível observar, parcialmente, o perfil de adoecimento no município (Ripsa, 2008). Os dados obtidos no Datasus revelam que as principais causas de internações nos seis municípios, exceto aquelas relacionadas à gravidez, parto e puerpério, são decorrentes de doenças do aparelho circulatório, do aparelho digestivo, do aparelho genito-urinário, do aparelho respiratório e envenenamentos ou outras consequências de causas externas, de acordo com a CID-10. A correlação das principais doenças atendidas na Atenção Básica e as plantas existentes fornecem informações que podem auxiliar na expansão das atividades com as plantas (Tabela 3 ).

Entre as 16 espécies nativas descritas na tabela 1, Maytenus ilicifolia Mart. ex Reissek (espinheira-santa) e Mikania glomerata Spreng. (guaco) já contam com resultados de pesquisas que atestam suas eficácia e segurança no tratamento de distúrbios digestivos e respiratórios (Graça

Rev. Bras. PI. Med., Campinas, v.17, n.3, p.398-406, 2015. 
TABELA 1. Espécies nativas citadas na RDC10/2010 e locais de cultivo / ocorrência na BHRV.

\begin{tabular}{|c|c|c|c|c|c|c|}
\hline FAMÍLIA / Espécie / Nome popular & $\mathrm{BH}$ & $\begin{array}{l}\text { C. Branco } \\
\text { Branco }\end{array}$ & Curvelo & Lassance & $\begin{array}{l}\text { N. } \\
\text { Lima }\end{array}$ & $\begin{array}{c}\text { S. } \\
\text { Lagoas }\end{array}$ \\
\hline \multicolumn{7}{|l|}{ ALISMATACEAE } \\
\hline $\begin{array}{l}\text { Echinodorus macrophyllus (Kunth) Micheli / Chapéu-de- } \\
\text { couro }\end{array}$ & $\mathrm{x}$ & & & & $\mathrm{x}$ & \\
\hline \multicolumn{7}{|l|}{ ANACARDIACEAE } \\
\hline Anacardium occidentale L. / Cajueirob & $x$ & & $x$ & & & \\
\hline \multicolumn{7}{|l|}{ ASTERACEAE } \\
\hline Ageratum conyzoides L. / Mentrasto & $x$ & $x$ & $x$ & & & $x$ \\
\hline Baccharis trimera (Less.) DC. I Carquejab & $x$ & $x$ & $x$ & $x$ & $x$ & $x$ \\
\hline Bidens pilosa L. I Picão ${ }^{b}$ & $x$ & $x$ & $x$ & & $x$ & $x$ \\
\hline Mikania glomerata Spreng. / Guacoa,b & $x$ & $x$ & $x$ & & $x$ & $x$ \\
\hline Vernonia polyanthes (Spreng.) Less. I Assa-peixe ${ }^{b}$ & $x$ & $x$ & $x$ & & $x$ & $x$ \\
\hline \multicolumn{7}{|l|}{ CELASTRACEAE } \\
\hline Maytenus ilicifolia Mart. ex Reissek / Espinheira santaa,b & $x$ & & $x$ & & & \\
\hline \multicolumn{7}{|l|}{ FABACEAE } \\
\hline Erythrina verna Vell. I Mulungu & $x$ & & $x$ & & & \\
\hline Stryphnodendron adstringens (Mart.) Coville / Barbatimão & $x$ & & $x$ & & & \\
\hline \multicolumn{7}{|l|}{ FLACOURTIACEAE } \\
\hline Casearia sylvestris Sw. / Guaçatonga ${ }^{b}$ & $x$ & & $x$ & & & \\
\hline \multicolumn{7}{|l|}{ MYRTACEAE } \\
\hline Eugenia uniflora L./ Pitangueirab & $x$ & & $x$ & & & \\
\hline \multicolumn{7}{|l|}{ PASSIFLORACEAE } \\
\hline Passiflora alata Curtis, P. edulis Sims / Maracujáb & $x$ & $x$ & $x$ & & $x$ & $x$ \\
\hline \multicolumn{7}{|l|}{ POLYGONACEAE } \\
\hline Polygonum punctatum Elliott / Erva-de-bicho & & & $x$ & & & $x$ \\
\hline \multicolumn{7}{|l|}{ SOLANACEAE } \\
\hline Solanum paniculatum L.I Jurubebab & $x$ & & $x$ & & & \\
\hline \multicolumn{7}{|l|}{ VERBENACEAE } \\
\hline $\begin{array}{l}\text { Lippia alba (Mill.) N.E. Br. ex Britton \& P. Wilson / Falsa } \\
\text { melissa }\end{array}$ & $\mathrm{x}$ & $\mathrm{x}$ & $\mathrm{x}$ & & $x$ & $\mathrm{x}$ \\
\hline
\end{tabular}

et al., 2007; Moura et al., 2002; Tavares et al., 2006). Essas duas espécies encontram-se, inclusive, incorporadas à lista de produtos com financiamento tripartite da assistência farmacêutica na atenção básica em saúde desde 2007 (Brasil, 2007). Segundo o levantamento epidemiológico, as doenças digestivas são as terceira e quinta causas de internações em Curvelo e Belo Horizonte, respectivamente, locais onde foi observada a produção da M. ilicifolia. Já o cultivo de Mikania glomerata Spreng. foi observado em cinco municípios (Belo Horizonte, Capim Branco, Curvelo, Nova Lima e Sete Lagoas), nos quais as doenças do aparelho respiratório aparecem como a terceira e/ou quarta causas de internação. Esses resultados revelam um potencial local de aproveitamento das duas espécies. Da mesma forma, onze espécies exóticas, descritas na tabela 2 , são frequentemente cultivadas em cinco dos seis locais estudados. Trata-se de espécies muito conhecidas e utilizadas em várias partes do mundo, sendo inclusive recomendadas pela OMS para uso como medicamento. Essas plantas produzem substâncias ativas contra várias doenças que causam internações na região estudada. $O$ estímulo à produção em larga escala das espécies listadas nas tabelas 1 e 2, já adaptadas à região, pode constituir uma forma de criação / expansão de negócios e fonte de renda para os produtores. A produção delas na região já revela a presença de condições favoráveis ao seu cultivo, em maior escala. Por outro lado, verifica-se que a qualidade do produto final depende de toda a

Rev. Bras. PI. Med., Campinas, v.17, n.3, p.398-406, 2015. 
TABELA 2. Espécies exóticas citadas na RDC10/2010 e locais de cultivo / ocorrência na BHRV.

\begin{tabular}{|c|c|c|c|c|c|c|}
\hline FAMÍLIAS / Espécies & $\mathrm{BH}$ & $\begin{array}{c}\text { C. Branco } \\
\text { Branco }\end{array}$ & Curvelo & Lassance & N. Lima & S. Lagoas \\
\hline \multicolumn{7}{|l|}{ APIACEAE } \\
\hline Pimpinella anisum L. / Erva-docea & $\mathrm{x}$ & $\mathrm{x}$ & $\mathrm{x}$ & & & $\mathrm{x}$ \\
\hline \multicolumn{7}{|l|}{ ASTERACEAE } \\
\hline Achillea millefolium L. / Mil folhas ${ }^{b}$ & $\mathrm{x}$ & & $x$ & & & \\
\hline Arctium lappa L. / Bardana & $\mathrm{x}$ & & $x$ & & & \\
\hline Calendula officinalis L. / Calêndula a,b & $\mathrm{x}$ & $x$ & $x$ & & & \\
\hline Taraxacum officinale F.H. Wigg. / Dente-de-leão & $x$ & & $x$ & & & \\
\hline \multicolumn{7}{|l|}{ LAMIACEAE } \\
\hline Melissa officinalis L. / Melissa a & $\mathrm{x}$ & & $\mathrm{x}$ & & & $x$ \\
\hline Mentha $x$ piperita L. / Hortelãs ${ }^{b}$ & $\mathrm{x}$ & $x$ & $x$ & & $x$ & $\mathrm{X}$ \\
\hline \multicolumn{7}{|l|}{ PLANTAGINACEAE } \\
\hline Plantago major L. / Trançagem ${ }^{\text {}}$ & $\mathrm{x}$ & & $x$ & & & \\
\hline \multicolumn{7}{|l|}{ POACEAE } \\
\hline Cymbopogon citratus (DC.) Stapf / Capim-santo & $x$ & & $x$ & & & \\
\hline \multicolumn{7}{|l|}{ PUNICACEAE } \\
\hline Punica granatum L. / Romãzeirab & & $x$ & $x$ & & & $x$ \\
\hline \multicolumn{7}{|l|}{ ZINGIBERACEAE } \\
\hline Zingiber officinale Roscoe / Gengibrea,b & $\mathrm{x}$ & $\mathrm{x}$ & $\mathrm{x}$ & & $\mathrm{x}$ & $x$ \\
\hline
\end{tabular}

$a=$ IN 05/2008, $d=$ Renisus (lista 71SUS)

cadeia produtiva envolvida, como cultivo, secagem e beneficiamento primário adequados, preparação e armazenamento dos fitoterápicos em conformidade com a legislação sanitária. Infelizmente, as fases anteriores ao processamento farmacêutico não possuem regulamentação específica; há apenas alguns manuais de Boas Práticas Agrícolas, desenvolvidos no âmbito governamental.

Em 2006, foi instituída a Política Nacional de Práticas Integrativas e Complementares no SUS, incluindo a Fitoterapia, a qual recomenda a Atenção Básica como local privilegiado para desenvolvimento de suas práticas (Brasil, 2006b). A perspectiva de prevenção de agravos e da promoção e recuperação da saúde desenvolvidas neste nível de atenção privilegiam o cuidado continuado, humanizado e integral em saúde. Ações como essas podem ser desenvolvidas pelas equipes da ESF. Os dados obtidos no Datasus mostram que a cobertura populacional de equipes da ESF no ano de 2010 nos municípios da BHRV são de $100 \%$ em 17 municípios, e de 80 a $99 \%$ em outros quatorze (Brasil, 2013a). Curiosamente, cinco municípios que contam com atividades de plantas medicinais possuem as menores coberturas da ESF: Belo Horizonte, Capim Branco e Curvelo contam com 60 a $80 \%$ de cobertura, enquanto em Nova Lima e Sete Lagoas a cobertura chega a menos de $45 \%$. Observa-se que as atividades com plantas medicinais estão desvinculadas da ESF, apesar de a população estar sensibilizada e haver demanda por tais recursos terapêuticos. Estímulos, como treinamento dos agentes de saúde, capacitação de profissionais de saúde e produtores e sensibilização de gestores, são necessários para a implantação da atividade desse setor.

A BHRV abrange dois biomas considerados mantenedores de elevada biodiversidade (Mata Atlântica e Cerrado, incluindo os campos de altitude), porém apenas $32,95 \%$ destes ainda se encontram preservados, enquanto $45,28 \%$ de toda a área da bacia já foi ocupada por atividades agropastoris. Além disto, boa parte do Rio das Velhas, juntamente a algumas de suas cabeceiras, está encaixada no Quadrilátero Ferrífero, sendo por isso uma das áreas mais críticas devido aos inúmeros empreendimentos de mineração (Polignano et al., 2001). A flora nativa vem também passando por intenso processo de erosão genética e cultural, que está levando ao desaparecimento de espécies com histórico importante na medicina tradicional (Brandão et al., 2009). Espécies medicinais importantes ocorrem de forma espontânea nos seis municípios estudados e algumas, como Baccharis trimera (Less.) DC. (carqueja), Stryphnodendron adstringens (Mart.) Coville (barbatimão), Echinodorus macrophyllus (Kunth) Micheli (chapéu-de-couro) e a Passiflora spp. (maracujá) contam com estudos que atestam suas eficácias e seguranças. O desenvolvimento de atividades locais que promovam o melhor aproveitamento destas e outras espécies da biodiversidade brasileira certamente contribuirá para 
TABELA 3. Principais causas de internações hospitalares, por local de residência, nos municípios estudados em 2010.

\begin{tabular}{|c|c|c|c|c|c|}
\hline \multirow[t]{2}{*}{ Município } & \multicolumn{5}{|c|}{ Causa de Internação } \\
\hline & $1^{a}$ & $2^{a}$ & $3^{a}$ & $4^{a}$ & $5^{a}$ \\
\hline $\begin{array}{c}\text { Belo } \\
\text { Horizonte }\end{array}$ & $\begin{array}{c}\text { Cap. XV: } \\
\text { Gravidez, parto } \\
\text { e puerpério }\end{array}$ & $\begin{array}{l}\text { Cap. IX: Doenças } \\
\text { do aparelho } \\
\text { circulatório }\end{array}$ & $\begin{array}{c}\text { Cap. XIX: Lesões, } \\
\text { envenenamentos e algumas } \\
\text { outras conseqüências de } \\
\text { causas externas }\end{array}$ & $\begin{array}{l}\text { Cap. X: Doenças do } \\
\text { aparelho respiratório }\end{array}$ & $\begin{array}{l}\text { Cap. XI: Doenças do } \\
\text { aparelho digestivo }\end{array}$ \\
\hline $\begin{array}{l}\text { Capim } \\
\text { Branco }\end{array}$ & $\begin{array}{c}\text { Cap. XV: } \\
\text { Gravidez, parto } \\
\text { e puerpério }\end{array}$ & $\begin{array}{l}\text { Cap. XIV: } \\
\text { Doenças } \\
\text { do aparelho } \\
\text { geniturinário }\end{array}$ & $\begin{array}{l}\text { Cap. IX: Doenças do } \\
\text { aparelho circulatório e Cap. } \\
\text { X: Doenças do aparelho } \\
\text { respiratório }\end{array}$ & $\begin{array}{l}\text { Cap. XI: Doenças do } \\
\text { aparelho digestivo }\end{array}$ & $\begin{array}{c}\text { Cap. XIX: Lesões, } \\
\text { envenenamentos e algumas } \\
\text { outras conseqüências de } \\
\text { causas externas }\end{array}$ \\
\hline Curvelo & $\begin{array}{c}\text { Cap. XV: } \\
\text { Gravidez, parto } \\
\text { e puerpério }\end{array}$ & $\begin{array}{c}\text { Cap. IX: Doenças } \\
\text { do aparelho } \\
\text { circulatório }\end{array}$ & $\begin{array}{l}\text { Cap. XI: Doenças do } \\
\text { aparelho digestivo }\end{array}$ & $\begin{array}{l}\text { Cap. X: Doenças do } \\
\text { aparelho respiratório }\end{array}$ & $\begin{array}{l}\text { Cap. XIV: Doenças do } \\
\text { aparelho geniturinário }\end{array}$ \\
\hline Lassance & $\begin{array}{c}\text { Cap. XV: } \\
\text { Gravidez, parto } \\
\text { e puerpério }\end{array}$ & $\begin{array}{l}\text { Cap. X: Doenças } \\
\text { do aparelho } \\
\text { respiratório }\end{array}$ & $\begin{array}{l}\text { Cap. XIV: Doenças do } \\
\text { aparelho geniturinário }\end{array}$ & $\begin{array}{l}\text { Cap. IX: Doenças do } \\
\text { aparelho circulatório }\end{array}$ & $\begin{array}{c}\text { Cap. XIX: Lesões, } \\
\text { envenenamentos e algumas } \\
\text { outras conseqüências de } \\
\text { causas externas }\end{array}$ \\
\hline Nova Lima & $\begin{array}{c}\text { Cap. XV: } \\
\text { Gravidez, parto } \\
\text { e puerpério }\end{array}$ & $\begin{array}{l}\text { Cap. IX: Doenças } \\
\text { do aparelho } \\
\text { circulatório }\end{array}$ & $\begin{array}{c}\text { Cap. XIX: Lesões, } \\
\text { envenenamentos e algumas } \\
\text { outras conseqüências de } \\
\text { causas externas }\end{array}$ & $\begin{array}{l}\text { Cap. X: Doenças do } \\
\text { aparelho respiratório }\end{array}$ & $\begin{array}{l}\text { Cap. XI: Doenças do } \\
\text { aparelho digestivo }\end{array}$ \\
\hline Sete Lagoas & $\begin{array}{c}\text { Cap. XV: } \\
\text { Gravidez, parto } \\
\text { e puerpério }\end{array}$ & $\begin{array}{l}\text { Cap. IX: Doenças } \\
\text { do aparelho } \\
\text { circulatório }\end{array}$ & $\begin{array}{l}\text { Cap. X: Doenças do } \\
\text { aparelho respiratório }\end{array}$ & $\begin{array}{c}\text { Cap. II: Neoplasmas } \\
\text { (tumores) }\end{array}$ & $\begin{array}{c}\text { Cap. XIX: Lesões, } \\
\text { envenenamentos e algumas } \\
\text { outras conseqüências de } \\
\text { causas externas }\end{array}$ \\
\hline
\end{tabular}

Fonte: Adaptado de Brasil, 2013b.

sua maior valorização e preservação. -

De acordo com Palos (2012), a articulação de diferentes instituições e atores no âmbito de um Arranjo Produtivo Local (APL) permite recuperar o conceito ampliado de saúde, a qual é resultante das condições de vida de uma dada sociedade educação, saúde, renda, trabalho, habitação, acesso e posse da terra, e que favorece a inclusão social, bem como a redução das desigualdades regionais e sociais e o uso sustentável da biodiversidade brasileira. O desenvolvimento da cadeia produtiva de plantas medicinais e fitoterápicos no âmbito de uma política social, como no caso da política de saúde do Brasil, possibilita a produção de tais insumos de forma a incrementar o acesso da população a esses recursos terapêuticos, conforme os princípios e diretrizes do SUS, e a estimular o desenvolvimento econômico e social local (Brasil, 2012). No entanto, a baixa cobertura populacional de equipes de Saúde da Família e a intensa erosão genética e cultural observadas nos cinco municípios constituem obstáculos a serem enfrentados para a ampliação das opções terapêuticas nestas localidades, e estruturação e consolidação da cadeia produtiva.
O apoio à estruturação e fortalecimento de Arranjos Produtivos Locais (APLs) em plantas medicinais e fitoterápicos no âmbito do SUS, iniciado recentemente pelo Ministério da Saúde, representa uma oportunidade interessante de superação dos referidos obstáculos. Em 2012 foram apoiados 14 projetos de Secretarias de Saúde das cinco regiões brasileiras: Betim (MG), João Monlevade / São Gonçalo do Rio Abaixo (MG), Botucatu (SP), Itapeva $(\mathrm{SP})$, Brejo da Madre de Deus (PE), Diorama (GO), Foz do Iguaçu (PR), Pato Bragado (PR), Toledo $(P R)$, Petrópolis (RJ), Rio de Janeiro (RJ), Santarém (PA), Alagoas e Rio Grande do Sul. O início de outros projetos, além da expansão dos dois APLs do estado de Minas Gerais, poderiam fomentar o trabalho colaborativo em rede e organizar a cadeia produtiva dos municípios em questão. Espera-se, em breve, que os resultados obtidos com tais projetos e a inovação gerada a partir das particularidades de cada região sirvam de base para a expansão da fitoterapia no SUS e a recolocação em cena da intersetorialidade e do conceito ampliado de saúde.

Rev. Bras. PI. Med., Campinas, v.17, n.3, p.398-406, 2015. 


\section{AGRADECIMENTO}

À FAPEMIG (Processo APQ 01912-10) pela

bolsa e apoio financeiro e ao Projeto Manuelzão pelos trabalhos de campo.

\section{REFERÊNCIAS}

BRANDÃO, M.G.L. et al. Qualidade de amostras comerciais de chás de plantas medicinais. Revista Brasileira de Plantas Medicinais. v.5, n.1, p.56-59, 2002.

BRANDÃO, M.G.L. et al. Traditional Uses of Brazilian Plants from the first Edition of Pharmacopoeia. Revista Brasileira de Farmacognosia, v.19, n.2a, p.478-87, 2009.

BRANDÃO, M.G.L. et al. Changes in the trade in native medicinal plants in Brazilian public markets. Environmental Monitoring and Assessment (print), v. 185, p.7013-7023, 2013.

BRASIL. Ministério da Saúde. Secretaria de Ciência, Tecnologia e Insumos Estratégicos. Departamento de Assistência Farmacêutica. Decreto 5.813, de 22 de junho de 2006. Dispõe sobre a Política Nacional de Plantas Medicinais e Fitoterápicos. Brasília: Ministério da Saúde, 2006a. 59p.

BRASIL. Ministério da Saúde. Secretaria de Atenção à Saúde. Departamento de Atenção Básica. Portaria $n^{\circ}$ 971, de 03 de maio de 2006. Dispõe sobre a Política Nacional de Práticas Integrativas e Complementares no SUS. Brasília: Ministério da Saúde, 2006b. 92p.

BRASIL. Ministério da Saúde. Portaria n 3.237, de 24 de dezembro de 2007. Aprova as normas de execução e financiamento da assistência farmacêutica na atenção básica em saúde. Diário Oficial [da] República Federativa do Brasil, Poder Executivo, Brasília, DF, 26 dez. 2007. Seção 1, p. 16.

BRASIL. Ministério da Saúde. Secretaria de Ciência, Tecnologia e Insumos Estratégicos. Departamento de Assistência Farmacêutica e Insumos Estratégicos. Portaria $n^{\circ} 2.960$, de 09 de dezembro de 2008. Aprova - Programa Nacional de Plantas Medicinais e Fitoterápicos. Brasília: Ministério da Saúde, 2008a. $136 p$.

BRASIL. Agência Nacional de Vigilância Sanitária. Instrução Normativa $n^{\circ} 05$, de 11 de dezembro de 2008. Determina a publicação da Lista de Medicamentos Fitoterápicos de Registro Simplificado. Diário Oficial [da] República Federativa do Brasil, Poder Executivo, Brasília, DF, 12 dez. 2008b. Seção 1, p. 56-58.

BRASIL. Ministério da Saúde. Lista de Plantas Medicinais de Interesse ao SUS (Renisus). 2009. Brasília. Disponível em: <http://portal.saude. gov.br/portal/saude/profissional/visualizar_texto. cfm?idtxt=30277>. Acesso em: 15 fev. 2013.

BRASIL. Agência Nacional de Vigilância Sanitária. Resolução de Diretoria Colegiada $n^{\circ} 10$, de 10 de março de 2010. Dispõe sobre a notificação de drogas vegetais junto à Agência Nacional de Vigilância Sanitária (Anvisa) e dá outras providências. Diário Oficial [da] República Federativa do Brasil, Poder Executivo, Brasília, DF, 10 mar. 2010. Seção 1, p. 52-54.
BRASIL. Ministério da Saúde. Secretaria de Ciência, Tecnologia e Insumos Estratégicos. Edital $\mathbf{n}^{\circ} 1$ SCTIE/MS para seleção de propostas de arranjos produtivos locais no âmbito do SUS, conforme a Política e o Programa Nacional de Plantas Medicinais e Fitoterápicos. 2012. Brasília. Disponível em: <http:// portal.saude.gov.br/portal/saude/profissional/visualizar texto.cfm?idtxt=40815\&janela=1>. Acesso em: $15 \mathrm{fev}$. 2013.

BRASIL. Ministério da Saúde. Secretaria de Gestão Estratégica e Participativa. Departamento de Informática do SUS - Datasus. Sala de Apoio à Gestão Estratégica. Disponível em: <http://datasus.saude. gov.br/informacoes-de-saude/business-intelligence-bi/ sala-de-apoio-a-gestao-estrategica-sage>. Acesso em: 15 fev. 2013a.

BRASIL. Ministério da Saúde. Secretaria de Gestão Estratégica e Participativa. Departamento de Informática do SUS - Datasus. Informações de Saúde (Tabnet) - epidemiológicas e morbidade. Disponível em: <http://www2.datasus.gov.br/DATASUS/index. php?area=0203>. Acesso em: 15 fev. 2013b.

CALIXTO, J.B. Twenty-five years of research on medicinal plants in Latin America. A personal view. Journal of Ethnopharmacology v.100, n.1-2, p.131-134, 2005.

GRAÇA, C. et al. In vivo assessment of safety and mechanisms underlying in vitro relaxation induced by Mikania laevigata Schultz Bip. ex Baker in the rat trachea. Journal of Ethnopharmacology, v.112, n.3, p.430-439, 2007.

INSTITUTO BRASILEIRO DE GEOGRAFIA E ESTATÍSTICA - IBGE. Censo 2010. Disponível em: <http://www.ibge.gov.br/home/estatistica/populacao/ censo2010/resultados_dou/default_resultados_dou. shtm>. Acesso em 20 fev. 2013.

MARTINS, E.M.L.P., BRANDÃO, M.G.L. Qualidade de amostras comerciais preparadas com Aesculus hippocastanum L. (castanha-da-Índia). Revista Brasileira de Farmacognosia, v.16, n.2, p.224-229, 2006.

MOURA, R.S. et al. Bronchodilator activity of Mikania glomerata Sprengel on human bronchi and guinea-pig trachea. Journal of Pharmacy and Pharmacology, v.54, n.2, p.249-256, 2002.

ORGANIZACÃO MUNDIAL DA SAUDE. UNICEF. Cuidados primários em saúde. In: Conferência Mundial sobre Cuidados Primários, 1979. Relatório final. Brasilia: Unicef, 1979. Disponível em: <http:// whqlibdoc.who.int/publications/9241800011_por.pdf>. Acesso em: 17 mai. 2012.

ORGANIZAÇÃO MUNDIAL DA SAÚDE. General guidelines for methodologies on research and evaluation of traditional medicine. Genebra, 2000. Disponível em: <http://apps.who.int/medicinedocs/ en/d/Jwhozip42e/>. Acesso em: $28 \mathrm{dez} .2011$.

ORGANIZAÇÃO MUNDIAL DA SAÚDE. The World Medicines Situation 2011: traditional medicines: global situation, issues and challenges. Genebra: Organização Mundial da Saúde, 2011. 12p.

PALOS, C.M.C. Avaliação e monitoramento das políticas de promoção de arranjos produtivos locais de plantas medicinais e fitoterápicos no Brasil. In: $1^{\circ}$ Seminário Regional de Arranjos Produtivos Locais de Plantas 
Medicinais e Fitoterápicos. 2012. Brasília. Disponível em: http://portal.saude.gov.br/portal/saude/profissional/ visualizar_texto.cfm?idtxt $=41579>$. Acesso em: $15 \mathrm{fev}$. 2013.

POLIGNANO, M.V. et al. Uma viagem ao projeto Manuelzão e à bacia do Rio das Velhas. Manuelzão vai à Escola. Belo Horizonte: Coleção Revitalizar, 2001. $64 p$.
REDE INTERAGENCIAL DE INFORMAÇÃO PARA A SAÚDE - RIPSA. Indicadores Básicos para a saúde no Brasil: conceitos e aplicações. 2 ed. Brasília: OPAS, 2008. 349p.

TAVARES, J.P. et al. Estudo de toxicologia clínica de um fitoterápico a base de associações de plantas, mel e própolis. Revista Brasileira de Farmacognosia, v.16, n.3, p.350-356, 2006. 